\title{
Duloxetine for the treatment of fibromyalgia
}

\author{
Cheryl L Wright ${ }^{\dagger, 1}$, Scott D Mist ${ }^{1}$, Rebecca L Ross ${ }^{1}$, and Kim D Jones ${ }^{1}$ \\ ${ }^{1}$ Oregon Health and Science University, School of Nursing, Portland, OR 97239, USA
}

\begin{abstract}
This article presents a brief review of the physiologic abnormalities seen in fibromyalgia, current theories of widespread pain, and treatment options, including emerging therapeutics, with a focus on the use of duloxetine to manage fibromyalgia symptoms. Major clinical trials that examine the efficacy and effectiveness of duloxetine to date are reviewed, and safety issues are discussed.
\end{abstract}

\section{Keywords}

duloxetine; fibromyalgia; pain; serotonin and norepinephrine inhibitors; SNRI

The purpose of this article is to discuss treatment options for fibromyalgia (FM), with a particular emphasis on the heterogeneity of treatment options and the role duloxetine may play in this population. A search was conducted using the following databases: PubMED, Medline Ovid and CINAHL using the search terms 'fibromyalgia', 'pain' and 'duloxetine'.

Fibromyalgia is a common, chronic and often debilitating syndrome characterized by muscle pain and stiffness, nonrestorative sleep and fatigue [1]. Many patients also experience a range of other somatic complaints that may include irritable bowel syndrome, cognitive impairment, headache, anxiety and depression, among others [2]. FM affects approximately 5 million people ( $1.6 \%$ of the population) in the USA, predominantly women [3], and results in significant economic [4-6], societal [7] and individual burdens [4,5]. For example, one survey of FM patients conducted at a US site reported that $70 \%$ perceived themselves as disabled, with $16 \%$ receiving Social Security benefits in comparison to $2.2 \%$ of the general US population [7]. People with FM also have higher lifetime and current rates of utilization of all types of medical services, with an average direct annual cost nearing US $\$ 10,000$ / patient/year, which translates into an estimated economic burden of over US\$20 billion annually [4]. For these reasons, medical management of symptoms is a high priority.

\section{Fibromyalgia diagnosis}

In 1990, the American College of Rheumatology (ACR) developed classification criteria for FM, which were formally established by a multicenter study. These criteria included a subjective report of widespread pain, above and below the waist, and on the left and right side of the body, including the axial spine. Furthermore, on physical examination, $4 \mathrm{~kg}$ or less of pressure applied slowly over $4 \mathrm{~s}$ to at least 11 out of 18 predefined tender points should elicit pain [8]. This formalization has allowed FM to become established as a legitimate clinical entity and has created subsequent research opportunities, which have led

\footnotetext{
(C) 2010 Expert Reviews Ltd

${ }^{\dagger}$ Author for correspondence: Tel.: +1 503494 3811, wrightch@ohsu.edu.

The authors have no other relevant affiliations or financial involvement with any organization or entity with a financial interest in or financial conflict with the subject matter or materials discussed in the manuscript apart from those disclosed.
} 
to an increased understanding of FM and other forms of chronic pain [9]. Recently, a revised diagnostic criterion was proposed [2]; however, the original 1990 criteria remains the gold standard with the most stringent criteria as well as commendable sensitivity and specificity [8].

To date, no single etiology of FM symptoms has been identified; however, in the past three decades, there has been progress in identifying physiologic abnormalities seen in patients with FM. Current theories underpinning the pathophysiology responsible for the increased pain and sensitivity in those with FM include abnormal sensitization from a peripheral pain stimulus, perhaps originating from deep muscle tissue or fascia [10,11], which may lead to sensitization of the CNS as a final common pathway for FM, as well as other chronic pain conditions [12]. This in turn may lead to more acute somatosensory perception or allodynia, a dysregulated autonomic nervous system [13], as well as altered CNS physiology [14-18]. Additional abnormal physiologic findings include abnormal sleep electroencephalography $[19,20]$, neuroendocrine perturbations [21], abnormal changes in the neurochemistry of cerebrospinal fluid suggestive of a proexcitatory state [22], cortical hyperactivation in response to both noxious and non-noxious stimuli $[10,14]$, disruptions in central dopaminergic neurotransmission $[23,24]$ and apparent acceleration in age-associated brain atrophy correlated with illness duration [25]. In addition, FM seems to aggregate in families, so a genetic predisposition seems likely. Several genes have been identified as possible candidates, principally those that involve neurotransmitter physiology [26].

As with all chronic illnesses, there is no known curative therapy for FM. At best, symptoms and functionality have been managed with varying degrees of success through a combination of pharmacological therapy [27], lifestyle changes [28] and complementary and alternative medicine therapies $[29,30]$.

\section{Current treatment guidelines}

Current FM treatment guidelines were well summarized in a recent review by Carville and colleagues, collaborators in the European League Against Rheumatism (EULAR) [28]. The group identified 146 research papers that offered the best evidence to support the most effective interventions for FM. Nine major conceptual and therapeutic realms were identified and included pharmacologic and nonpharmacologic approaches, ideally in the form of a comprehensive multimodal approach. Recommendations included the healthcare provider's adequate understanding of the complexity and heterogeneity of FM. Providers should understand that FM is a chronic pain syndrome, with abnormal pain processing and associated features. In addition, the provider should perform a comprehensive evaluation of pain, function and psychosocial coping. Finally, the provider must offer symptom-specific pharmacological and nonpharmacological interventions, with close attention paid to individual symptoms and subsequent response to treatment plans, and be willing to make adjustments as necessary [28].

There are also evidence-based nonpharmacological interventions that deserve consideration. These include balneotherapy in heated pools with or without exercise [31], individually tailored exercise programs with both aerobic and anaerobic exercises [32], cognitive behavioral therapy [33], relaxation/meditation, formal rehabilitation and/or physical therapy [34] and, while still controversial, acupuncture [35,36]. Less conventional interventions that could be considered include biofeedback [37], heart rate variability feedback [38] and transcranial direct current stimulation $[39,40]$. 


\section{Current FM pharmacotherapy}

Currently, there is a wide range of pharmacologic agents frequently used alone or in combination to manage FM symptoms, however, only three are formally indicated by the US FDA for FM: two selective serotonin and norepinephrine reuptake inhibitors (SNRIs), duloxetine and milnacipran, and pregabalin, an anticonvulsant. Despite lack of formal approval, patients and prescribers use other, unapproved agents with varying degrees of success to manage FM symptoms. Some of these agents include nonsteroidal antiinflammatory drugs (NSAIDs), acetaminophen, opioids, selective serotonin reuptake inhibitors (SSRIs), antispasmodics, dopamine agonists, hypnotics, benzodiazepines and/or muscle relaxants [27]. However, only four of the aforementioned pharmaceutical agents have multicenter research-backed clinical trial evidence to support their use: amitriptyline [41], pregabalin [42], milnacipran [43] and duloxetine, the focus of this article.

\section{Mechanism of action}

For widespread chronic pain conditions such as FM, the mechanism of action for SNRIs is not fully understood, but is thought to be directly related to serotonin and norepinephrine reuptake inhibition at the level of the dorsal horn in descending pain pathways. The triad of chronic pain, depression and anxiety that lead to inter-related symptoms is well described, but our evolving understanding of neurophysiology in chronic pain, while not clear, suggests a direct role for neurotransmitters in analgesia [44,45]. This is best illustrated with Melzack's (1999) theory of chronic pain, which involves complex physiological and psychological interplay - a neuromatrix model that involves a network-type of functioning in the CNS that involves the nerves as well as the neurotransmitters, hormones and receptors, rather than any single structure or physiologic response to painful stimuli [46]. The neuromatrix theory is bolstered by CNS imaging studies that suggest CNS neural networks, rather than any single region, are engaged in those with chronic pain [15,17]. The characteristic widespread pain and decreased pressure pain thresholds (e.g., increased sensitivity to the light touch of a child or a hug) experienced with FM is thought to be multifactorial and may include altered hypothalamic-pituitary axis chemistry [47], abnormal levels of corticotropin-releasing factor in cerebrospinal fluid [48], abnormal $N$-methyl Daspartate and neurokinin-1 receptors in the dorsal horn [49] and, finally, emotional affective response to pain [50]. Importantly, the analgesic effect associated with SNRIs has been demonstrated to be independent of effect on mood [51-53].

\section{Overview of the market}

To date, managing the chronic pain of FM has represented a major challenge for healthcare providers [54]. FM studies suggest that chronic pain management responds best to multidisciplinary approaches [28,54], and from these models, strategic uses of pharmaceuticals play an important role. In general, regular use of opioids is discouraged for chronic pain management as they are generally not as effective as other agents and may lead to a paradoxical hyperalgesia in a subset of patients, which may in part be due to a saturation of opioid receptors from endogenous opioids [55]. In addition, in subjects with FM, positron emission tomography suggests that $\mu$-opioid binding potential is significantly decreased in regions responsible for pain moderation, specifically the nucleus accumbens, dorsal cingulate and amygdala, which suggests a mechanism for the clinically observed poor response to opioids in FM [56]. Finally, common opioid side effects such as constipation, drowsiness and tolerance usually result in their limited role for chronic pain [55,57].

NSAIDs alone often fail to relieve chronic muscle pain of FM [27], but may be useful in reducing peripheral pain from regional pain generators, such as bursitis, tendonitis or plantar fasciitis, as an adjunct therapy. 
Currently, the mainstays of pharmacological management for FM include the tricyclic class of antidepressants (amitriptyline), weak $\mu$-opioid agents (tramadol), SNRIs (duloxetine and milnacipran) and one anticonvulsant (pregabalin) [27]. Unfortunately, all studies of these agents in FM to date have been washout/monotherapy studies. In clinical practice, providers often combine pharmacologic agents based on rational mechanisms of action without the benefit of empiric data. One small-scale study illustrates this point. Zucker et al. conducted a practice-based study where individuals were studied instead of groups in what is known as an N-of-1 trial. Zucker and colleagues examined $58 \mathrm{~N}$-of-1 trials designed to look at combination therapy for FM, using amitriptyline alone or in combination with fluoxetine. The results illustrate a practice-based research design that may more accurately reflect clinical practice, while also examining the question of best patient outcomes. Currently, we know that noradrenaline reuptake has better antinociceptive qualities than serotonin reuptake alone; however, these study results are important for methods as most patients suffering with chronic pain will not be on monotherapy [58].

\section{Competitor compounds that are in clinical or late-phase development}

There are four emerging therapies on the horizon for FM symptom management: sodium oxybate (Xyrem®, Jazz Pharmaceuticals, CA, USA), flupirtine, lacosamide (Vimpat®, Schwarz Pharma, CO, USA) and low-dose naltrexone. Sodium oxybate is a schedule III compound approved by the FDA for the treatment of cataplexy and excessive daytime sleepiness. Recently, in Phase III FM trials, sodium oxybate was shown to improve sleep and other FM symptoms including pain and fatigue [59]. Sodium oxybate is currently under review by the FDA for a FM indication based on multisite randomized controlled clinical trials in adults with FM [59]. Unfortunately, owing to the lack of current FDA indication for FM, many practitioners are unaware of its potential benefit in this population.

Flupirtine is an aminopyridine that has been used successfully since 1984 in many European countries for acute and chronic pain. After positive results from a serendipitous case series of four FM patients [60], Stoll and colleagues were approved to begin a Phase II study in 2008 to examine flupirtine's effectiveness for FM symptoms [61,101]. Lacosamide, an anticonvulsant with a mechanism of action related to sodium channel inactivation, which is not yet FDA approved for FM, may be approved in the USA and Europe in late 2010. A recent Phase II trial concluded that lacosamide was effective and well tolerated in a sample of patients with diabetic peripheral neuropathic pain [62], results of which may translate to a FM population. Finally, two small studies suggest effective pain relief in FM samples using low-dose naltrexone (LDN) dosed at $4.5 \mathrm{mg}$ per day. The potential mechanism of action hypothesized for LDN is a reduction of proinflammatory cytokines and neurotoxic superoxides through an action on Toll-like receptors [63], as a separate study with 50-mg naltrexone per day suggested the mechanism of pain was not directly due to abnormal endogenous opioid activity [64]. In addition to the single small pilot study, a larger clinical trial to examine LDN for FM is currently ongoing in the USA (NCT00568555).

\section{Introduction to duloxetine}

Duloxetine is a SNRI that has been approved for use by the FDA for maintenance and management of acute major depressive disorder (MDD), generalized anxiety disorder (GAD), diabetic peripheral neuropathic pain (DPNP) and FM [102].

\section{Chemistry}

The chemical name of duloxetine is (+)-(S)- $N$-methyl- $\gamma$-(1-naphthyloxy)-2thiophenepropylamine hydrochloride. The empirical formula is $\mathrm{C}_{18} \mathrm{H}_{19} \mathrm{NOS} . \mathrm{HCl}$ and the molecular weight is $333.88 \mathrm{~g} / \mathrm{mol}$ [65]. Duloxetine is a white to slightly brownish powder 
and is slightly water soluble. Capsules are dispensed in doses of 20,30 and $60 \mathrm{mg}$, and are enteric coated to prevent dissolution in the stomach, which allows for slower release times [103].

\section{Pharmacodynamics}

While an accurate biomarker for CNS norepinephrine reuptake is elusive, there is convincing clinical evidence that duloxetine is a potent and balanced reuptake inhibitor of serotonin and norepinephrine [66]. There is no evidence of significant effects on monoamine oxidase inhibition, dopaminergic, adrenergic, cholinergic, histaminic, muscarinic, opioid, glutamate or GABA receptors in vitro [103].

Duloxetine is metabolized by the CYP450 isoenzymes CYP1A2 and CYP2D6 [67]. As it is a potent CYP1A2 inhibitor, duloxetine should be used cautiously with concurrent use of cimetidine and quinolone antibiotics such as ciprofloxacin and enoxacin [68]. For those who are CYP2D6-poor metabolizers, duloxetine may be excreted more slowly, resulting in up to six-fold higher serum levels [67]. Concurrent smoking may decrease duloxetine bioavailability by approximately $30 \%$; however, dose adjustment is not recommended [69]. Duloxetine is not recommended in those with hepatic or severe renal insufficiency, or in those with heavy alcohol use, due to the risk of liver damage, although ongoing monitoring of liver enzymes is not required for patients without known liver disease [70]. Duloxetine may have up to $30 \%$ less bioavailability in men than women, putatively due to the increased CYP1A2 metabolism [69], and is excreted in breast milk at a rate of approximately $25 \%$ of maternal serum levels [71]. There is no evidence that ethnicity affects duloxetine metabolism [72,73].

\section{Pharmacokinetics \& metabolism}

Duloxetine has an elimination half-life of $12 \mathrm{~h}$, but this can range between 8 and $17 \mathrm{~h}$. Pharmacokinetics are dose proportional over the therapeutic range, and plasma concentrations reach steady state in approximately 3 days [68], with a primarily hepatic elimination [67]. It is well absorbed upon oral administration with a median 2-h delay before absorption begins, and peak plasma concentrations are reached $6 \mathrm{~h}$ after dose administration [65]. When taken with food, peak plasma concentrations are not affected, but time to peak is slightly delayed to $10 \mathrm{~h}$, with absorption minimally decreased by $10 \%$. When taken in the evening instead of the morning, absorption is delayed by $3 \mathrm{~h}$ along with a 33\% increase in clearance time [103].

Duloxetine is extensively metabolized and major metabolites have not been shown to contribute to the pharmacological effects. Less than $1 \%$ of duloxetine is excreted unchanged in the urine, approximately $70 \%$ is excreted in urine as metabolites and approximately $20 \%$ is excreted in the feces, also as metabolites [74].

Duloxetine has a bioavailability of approximately $50 \%$ with a range of $32-80 \%$, depending on the time of day and fasting status, and it is significantly protein bound at approximately $95 \%$ [103]. Because duloxetine is so highly bound to plasma protein, administration in those concurrently taking other highly protein-bound drugs may cause increased free concentrations of either drug, with potential adverse reactions [103].

\section{Clinical efficacy}

\section{Phase I \& II studies}

Prior animal and human studies were performed as part of duloxetine's clinical development in accordance with FDA requirements [102]. Metabolism, excretion and pharmacokinetics 
of duloxetine were first established in healthy human subjects [65,74]. Duloxetine received FDA indications for the treatment of MDD, DPNP and GAD prior to its approval for FM in 2008 [102]. In addition to the multiple Phase III clinical trials performed to establish safety and efficacy for the aforementioned indications, additional studies were conducted in people with FM.

\section{Phase III studies}

The efficacy and safety of duloxetine for the treatment of FM was investigated in the USA in four short-term studies [51,75-77] and one long-term open-label study [78], primarily by one group of researchers, the Duloxetine Fibromyalgia Trial Group (see Table 1). In each study, subjects were randomly assigned to various fixed dosages of duloxetine that ranged from $60 \mathrm{mg}$ per day to $120 \mathrm{mg}$ per day.

The first major study examined the effects of duloxetine for pain in FM subjects who met ACR criteria for FM $[8,75]$. Subjects randomized to receive duloxetine initially underwent a double-blind forced titration from $20 \mathrm{mg} /$ day up to $60 \mathrm{mg}$ twice daily during the first 2 weeks of the therapy phase. The dose received during the study was $60 \mathrm{mg}$ twice daily. Arnold and colleagues (2004) had two primary outcome measures, the Fibromyalgia Impact Questionnaire (FIQ) total score, and the FIQ single-item pain score measured on a ten-point visual analog scale (VAS). The sample size was powered to detect a 1.4 difference on the FIQ pain score and was not powered to detect a significant change in the FIQ total score.

Secondary outcome measures included average tender point pain thresholds, number of tender points, FIQ fatigue, tiredness upon awakening and stiffness scores, Clinical Global Impression of Severity (CGI-S) scale, Patient Global Impression of Improvement (PGI-I) scale, Brief Pain Inventory (BPI; short form), Medical Outcomes Study Short Form 36, Quality of Life in Depression scale and the Sheehan Disability Scale (SDS). Because a secondary goal of the study was to determine if pain relief was related to amelioration of depressive or anxiety symptoms that would also respond to SRNI therapy, two measures were collected as secondary outcome measures: the Beck Depression Inventory-II and the Beck Anxiety Inventory.

Notable results that emerged from this study included significant response differences to duloxetine between the genders. In addition, among the female subjects, those who received duloxetine improved significantly more than placebo-treated women on all of the secondary efficacy measures, while the men who received duloxetine did not respond significantly on primary or secondary efficacy measures. Furthermore, there were no interactions between depression scores and pain improvement, suggesting that pain relief is not dependent upon depression improvement.

Building on the results from the previous trial, the second major study was also undertaken by Arnold and colleagues [51], however, only women who met ACR criteria for FM were enrolled. Subjects were randomized in a 1:1:1 ratio to one of three treatment groups: duloxetine $60 \mathrm{mg}$ once daily, duloxetine $60 \mathrm{mg}$ twice daily (repeating the aforementioned strategy of forced titration from $60 \mathrm{mg}$ once daily for 3 days to $60 \mathrm{mg}$ twice daily) or placebo. In this second study, the primary outcome measure was the average pain score from the BPI, which captures self-reported pain during the past $24 \mathrm{~h}$, and secondary outcome measures were similar to the prior study with the exception of the Hamilton Depression Rating Scale. This study was powered to detect a group change of -1.2 points on the BPI average pain score. The results from this second trial found that both duloxetine $60 \mathrm{mg}$ once daily and twice daily significantly reduced the 24 -h average pain score of the BPI, and again, no interaction between depression and pain improvements was noted. One additional important finding was that duloxetine provided clinically relevant pain relief, defined as the 
percentage of subjects who experienced a $30 \%$ or greater reduction in pain measured with the BPI. Specifically, the percentage of subjects who achieved at least a $30 \%$ improvement was statistically significant compared with placebo using both the a priori ana lysis of the last observation carried forward as well as the more stringent baseline observation carried forward ana lysis. In addition, a statistically significant percentage of subjects achieved the higher goal of $50 \%$ improvement for duloxetine $60 \mathrm{mg}$ once daily compared with placebo. This is an important finding because previously a 30\% reduction in pain has been considered to be clinically meaningful [79]. Recently, however, many experts involved in the management of DPNP have raised the bar and recommended that researchers adopt a 50\% reduction in pain as a more appropriate treatment goal [80]. In addition to the BPI outcome, the mean change in the FIQ total score from baseline to end point was reduced to 16.72 for duloxetine compared with only $8.35(\mathrm{p} \leq 0.05)$ for the placebo group, which suggests that duloxetine $60 \mathrm{mg}$ once daily was also able to significantly improve function [51].

The third study was multisite, conducted by the same group of researchers [76], with the $a$ priori goal to determine the most effective duloxetine dose required for improved pain scores, measured with the average score on the BPI short form and the PCG-I. This study compared three daily dosage regimens, 20,60 and $120 \mathrm{mg}$, to placebo over 6 months in male and female subjects who met ACR criteria for FM, however $94.8 \%$ of the sample were female. Subjects were randomized to treatment groups in a 1:2:2:2 ratio, respectively. Subjects on more than $20 \mathrm{mg} /$ day were were titrated up and off study medication. At 3 months, the $20-\mathrm{mg} /$ day group was titrated up to $60 \mathrm{mg} /$ day for the remaining 3 months.

Two primary outcomes included the average score of the BPI short form and the PCG-I, or subject's perception of improvement. Secondary outcomes were similar to the two prior studies [51,75] with the addition of the Multidimensional Fatigue Inventory (MFI) and the European Quality of Life Questionnaire-5 dimensions. The study was powered to detect a -1.2 point-change on the BPI average pain score and a 0.68 -point change in the PCG-I between duloxetine $120 \mathrm{mg} /$ day and placebo. Subjects who completed this 6-month study were eligible to participate in a 6-month extension phase in which all subjects received 120 $\mathrm{mg}$ of duloxetine once daily.

Important findings from this study included a significance in dose-related discontinuation rates due to adverse events associated with $120 \mathrm{mg} /$ day and, while not reaching statistical significance, a discontinuation rate in the 20-mg/day group due to lack of effectiveness. In contrast to prior findings [51], the 50\% improvement goal was not significant for the 20- or $60-\mathrm{mg} /$ day group, but was met in $40.1 \%$ of the $120-\mathrm{mg}$ /day group compared with placebo (p $=0.003)$. The number needed to treat for each dosing regimen of 20,60 and $120 \mathrm{mg} /$ day was 12,10 and 7 , respectively.

In the fourth study by Chappell and colleagues [77], subjects were randomly assigned to 23 weeks of duloxetine treatment ( 8 weeks at $60 \mathrm{mg}$ once daily followed by 15 weeks of duloxetine continued at $60 \mathrm{mg}$ once daily or increased to $120 \mathrm{mg}$ once daily depending on clinical response and tolerability, or placebo). All subjects who completed the initial therapy phase in study four were eligible to enter a 29-week double-blind extension phase at either 60 or $120 \mathrm{mg}$ once daily, depending on dose at the end of the short-term therapy phase. Primary outcome measures, similar to prior studies, included the BPI average pain score plus the PGI-I. Results of these primary outcome measures were not statistically significant in the duloxetine group compared with the placebo group. However, several secondary outcome measures, such as FIQ, CGI-S and the MFI, did show statistically significant improvement. 
In addition to efficacy, the goal of the fifth study was to establish the long-term safety of duloxetine for the treatment of FM [78]. This was a multisite, international study where male and female subjects were recruited, but similar to previous studies, $95.7 \%$ of subjects were female. Initially open label, after a lead-in period of 7 days on 30-mg duloxetine/day, the dose was titrated up to $60 \mathrm{mg} /$ day for 7 weeks. Subjects and researchers were subsequently blinded and subjects were randomized to duloxetine $60 \mathrm{mg}$ or $120 \mathrm{mg} /$ day in a 1:2 ratio, for the next 52 weeks. This study was powered to assess adverse events in 100 subjects assigned to $120 \mathrm{mg} /$ day, and not powered for improvement in other outcome measures, although response status was measured with the BPI average pain score and responders were defined as those who achieved $50 \%$ or more improvement on the BPI during the 8-week open-label phase. Subjects were titrated up and off study medication. Efficacy outcomes were measured using the BPI modified short form that captured severity and interference scores, in addition to the FIQ, CGI-S, PGI-I, SDS and Global Functioning Impairment Scale, as well as mean pain threshold and number of tender points. In addition to these outcomes, the primary outcomes of safety were measured frequently and included queries regarding adverse events, concomitant therapies, vital signs, urinalysis panels, blood chemistry panels and hematology. For screening purposes, pregnancy tests, urine drug screening tests for illicit drugs and electrocardiograms were performed.

All analyses were based upon intent-to-treat, and all subject data were analyzed for safety. Drug safety was the primary outcome measure. As such, $22.7 \%$ of the sample discontinued duloxetine due to adverse events, which included insomnia, vomiting, diarrhea, dizziness and nausea. At least 10\% or more of the sample reported nausea, headache, insomnia, dizziness, constipation, dry mouth, somnolence, diarrhea, fatigue, hyperhidrosis and abdominal pain. During the open-label phase, $81.7 \%$ of subjects experienced at least one treatment-emergent adverse event, while during the double-blind phase, $85.6 \%$ of subjects on $60 \mathrm{mg} /$ day and $88.2 \%$ of subjects on $120 \mathrm{mg} /$ day experienced a treatment-emergent adverse event, with 13.5 and $16.7 \%$, respectively, discontinuing treatment due to an adverse event.

Although clinically insignificant, treatment emergent abnormal laboratory values included 8.9\% with elevated alkaline phosphatase, $18.6 \%$ with elevated alanine aminotransferase/ serum glutamate pyruvate transaminase, $11.8 \%$ with elevated aspartate aminotransferase/ serum glutamic oxaloacetic transaminase, $9.2 \%$ with elevated cholesterol, $9.2 \%$ with elevated creatinine phosphokinase, $7.5 \%$ with elevated $\gamma$-glutamyl transferase (GGT) and $5 \%$ with elevated urea nitrogen. A total of 5\% developed low serum potassium. However, values thought to be clinically significant included cholesterol (2.1\%), and GGT in two subjects, one male (195.0 U/l) and one female (135.0 U/l). Overall, the safety of long-term treatment with duloxetine at 60 or $120 \mathrm{mg} /$ day was deemed satisfactory.

Secondary outcome results indicated that during the open-label phase, $34.8 \%$ of the sample could be classified as 'responders', which meant that they reported greater than 50\% pain improvement. During the second double-blind phase, results were mixed, but suggest that at 60 or $120 \mathrm{mg} / \mathrm{day}$, subjects reported perceptions of overall improved condition and function, and specifically, improved walking ability, improved sleep and fewer trigger points with lower trigger point pain thresholds. Particularly salient results from this study of long-term safety suggest that not only were there no significant increased benefits at $120 \mathrm{mg} / \mathrm{day}$ as opposed to $60 \mathrm{mg} / \mathrm{day}$, but actually there was a higher incidence of adverse reactions.

Subsequent to the aforementioned five clinical trials, there have been five additional post hoc analyses or reviews, including a Cochrane review that examined the pooled results of some or all of these trials [70,81-84]. In general, findings have been positive, and suggest that duloxetine $60 \mathrm{mg} /$ day is safe [70] and effective for controlling pain in those with FM 
[81-83], yet may not be more effective than other antidepressants on the market to control FM pain [84].

Despite the evidence for safety and effectiveness $[51,70,75-77,81-84]$ there remains some controversy around the safety and efficacy of duloxetine to treat FM pain. An anonymous author for Prescrire International wrote the following with regard to the use of duloxetine for depression and diabetic neuropathy: "Duloxetine offers no therapeutic advantage in treating either condition [depression or diabetic neuropathy]. There is no reason to choose duloxetine when so many other options are available" [85]. By contrast, in 2008, The Medical Letter on Drugs and Therapeutics found that despite some 'troublesome adverse effects and drug interactions' duloxetine was effective for FM symptom management [86]. However, another author questions industry bias and argues that the analgesic properties of duloxetine have been overstated. Speilmans' independent ana lysis using a random effects model suggested a small effect size with a nonsignificant analgesic effect [87].

\section{Post-marketing surveillance}

From 2004 through to 2006, FDA post-marketing incidents of duloxetine-associated adverse events include hyponatremia, falls, abnormal bleeding with an increased prothrombin time/ international normalized ratio, urinary hesitancy and liver toxicity [104]. Additional reports include incidents of anaphylactic shock, inappropriate aggression or anger - especially during the early treatment phase - angioneurotic edema, erythema multiforme, extrapyramidal disorder, glaucoma, gynecological bleeding, hallucinations, hyperglycemia, hypersensitivity, hypertensive crisis, muscle spasm, rash, restless leg syndrome, seizures after treatment discontinuation, supraventricular arrhythmia, tinnitus (upon treatment discontinuation), trismus and urticaria. Serious skin disorders including Stevens-Johnson syndrome have also been reported [65].

In July 2006, the FDA released an alert regarding the potentially life-threatening serotonin syndrome with combined use of SSRIs or SNRIs and triptan medications. The FDA reviewed 27 reports of serotonin syndrome reported in association with concomitant SSRI or SNRI and triptan use. Two reports described life-threatening events and 13 reports stated that the patients required hospitalization. Some of the cases occurred in patients who had previously used concomitant SSRIs or SNRIs and triptans without experiencing serotonin syndrome. The reported signs and symptoms of serotonin syndrome were highly variable and included respiratory failure, coma, mania, hallucinations, confusion, dizziness, hyperthermia, hypertension, sweating, trembling, weakness and ataxia. In eight cases, recent dose increases or the addition of another serotonergic drug to an SSRI/triptan or SNRI/ triptan combination were temporally related to symptom onset. The median time to onset subsequent to the addition of another serotonergic drug or dose increase of a serotonergic drug was 1 day, with a range of 10 min to 6 days $[88,105]$.

\section{Safety \& tolerability}

In animal models, at recommended human doses, there was no evidence of carcinogenesis, mutagenesis or impaired fertility [103]. Duloxetine should be used with caution in people with controlled narrow-angle glaucoma, hepatic disease, alcohol abuse or severe renal insufficiency (creatinine clearance $<30 \mathrm{ml} / \mathrm{min}$ ), and may lead to worsened glycemic control in diabetics (mean hemoglobin A1c increased by $0.5 \%$ compared with the routine care group, which increased by $0.2 \%$ ) [103].

In clinical studies, side effects were dose related and included nausea, headache, dry mouth, fatigue/asthenia, insomnia, dizziness, somnolence/hypersomnia/sedation, constipation, diarrhea and decreased appetite (see $\mathbf{F}_{\text {Igures }} 1 \&$ 2). Nausea, somnolence and fatigue were 
reported as a rationale for discontinuation in 1.9. 1.5 and 1.3\% of pooled FM study subjects, respectively [70]. While these side effects tended to diminish over time [78], in one population-based study, $56 \%$ of women stopped therapy due to side effects and $33 \%$ due to lack of efficacy [73]. Despite earlier concerns, there were no appreciable cardiovascular effects, including clinically significant increases in blood pressure [89], and the FDA has not recommended specific laboratory monitoring [103].

A second area of caution includes platelet release of serotonin and hemostasis [90]. There is some evidence of an association between psychotropic agents that affect the reuptake of serotonin and upper gastrointestinal bleeding in those who are concurrently using NSAIDs such as aspirin or ibuprofen [91]. There have also been reports of altered anticoagulation effects in those who are anticoagulated with warfarin [92], so close monitoring of these individuals is warranted with duloxetine initiation or discontinuation [103]. Duloxetine does not affect CYP2C9 or CYP3A, thus the therapeutic effects of oral contraceptives and other steroidal agents are not compromised [103].

Like all antidepressants, duloxetine has a boxed warning for suicidality. Specifically, children and young adults up to the age of 24 years have an increased risk of suicidality with anti depressants compared with placebo [93], but in the first months of SSRI therapy, depressed geriatric patients also have an increased risk of suicide [94]. In addition, activation of mania/hypomania is possible when using an SSRI or SNRI. Primary care providers or rheumatologists may not recognize the early signs of type II bipolar disorder in their FM patients [95], but should be alert to this possible reaction, and speciality providers such as rheumatologists, compared with psychiatrists, may not be skilled at how to best elicit a history of hypomania.

\section{Regulatory affairs}

Currently, the FDA has approved duloxetine for use in four conditions: MDD (2004), DPNP (2004), GAD (2007) and FM (2008) [102]. In addition to these, the EU approved duloxetine for the treatment of stress urinary incontinence in 2004.

\section{Conclusion}

While additional nonindustry-sponsored investigation is warranted, the evidence to date suggests that in those with FM, duloxetine has largely been a safe and, in some, a promising intervention to manage chronic pain. There have been a fair number of uncomfortable side effects reported, which seem to attenuate over 4-6 weeks. Specific to the heterogeneous symptoms that accompany FM, duloxetine may effectively diminish pain and increase physical function in some, although unsurprisingly, not in all. As with the introduction of any new pharmacologic agent, each patient deserves close monitoring to assess the drug's effectiveness as well as adverse effects.

\section{Expert commentary}

During the past 8 years, duloxetine has been available for clinical use for a variety of disorders including depression and anxiety, as well as painful conditions such as FM and DPNP. Duloxetine was the first SNRI to be FDA indicated for FM. Prior to duloxetine, only one other agent, pregabalin, had been indicated for the treatment of FM. FM awareness programs, funded in part by the pharmaceutical industry, have raised patient and provider expectations for treatment options in FM.

Prior to FDA-indicated medications for FM, providers relied heavily on tricyclics, muscle relaxants, opioids, tramadol and SSRIs. The tricyclic studies in FM, while positive, were 
limited by side effects, which usually occurred during long-term use that incorporated dose escalation, and included effects such as dry eyes/mouth, constipation, weight gain and potentially serious QT changes, particularly in older patients. Therefore, while tricyclics were widely prescribed, adherence was generally poor. Muscle relaxants and NSAIDS, unfortunately, proved negative in clinical trials for FM, yet their use in FM persists, perhaps, in the case of NSAIDs, due to over-the-counter availability. Tramadol, both short and long acting, is indicated for pain rather than FM symptom management, despite an FDA review of two positive multicenter trials of tramadol in a FM sample. Therefore, prescribers may be less aware of the potential role tramadol may have in the management of FM symptoms. Opioids, while viewed favorably by many FM patients, are hampered by a lack of prospective trials in FM samples, as well as provider concerns about dependence, physiologic side effects or diversion. Finally, one of the most prescribed classes of drugs for FM patients are the SSRIs. However, as SSRIs have become increasingly selective for serotonin reuptake, their efficacy in FM trials has declined; in fact, they are negative. Perhaps providers continue to prescribe SSRIs in FM due to concerns about possible concomitant depression. Providers may lack awareness that SNRIs such as duloxetine have been demonstrated to improve pain, global FM symptoms, anxiety and depression. This combination makes duloxetine a reasonable choice for many patients with FM.

In terms of combination therapies, duloxetine is thought to exert its analgesic effect by manipulation of serotonin and norepinephrine reuptake in descending pain pathways at the level of the dorsal horn. Anticonvulsants, such as gabapentin and pregabalin, are thought to reduce FM pain in ascending pathways by limiting the release of excitatory

neurotransmitters through voltage-gated calcium channels at the $\alpha-2 \delta$ subunit. Although to date there are no published multisite combination studies of duloxetine and gabapentin or pregabalin, many providers prescribe them in concert due to the theoretical effect on complementary aspects of aberrant pain processing. Finally, duloxetine is the only FDAindicated agent with once-daily dosing. It is weight neutral and exerts a minimal effect on pulse and blood pressure ( $2.3 \mathrm{mmHg}$ mean diastolic increase). Its side effect of increased urethral resistance may be viewed positively by the $30-40 \%$ of FM patients with irritable/ painful bladder and frequent urination. Interestingly, while no agents are indicated for FM in Europe at the time of writing, duloxetine is indicated for treating stress urinary incontinence in Europe.

As mentioned earlier, concerns about duloxetine largely revolve around its metabolism and utilization of the CYP 2D6 enzyme, a commonly used pathway. The average FM patient is on between three and six medications for FM symptoms alone, thus there may be increased risks for drug-drug interactions. However, when the data are taken as a whole, the benefits of duloxetine outweigh the risk for many patients.

Generally speaking, FM patients present on average with 16 or more symptoms, making medical management decisions difficult. A skilled provider can improve his/her treatment efficacy by tracking individual patient progress using a tool specifically designed to track FM symptom improvements. One excellent example of this is the Fibromyalgia Impact Questionnaire-Revised (FIQR), available for general use, without charge at [106]. Using a patient self-administered tool like the FIQ-R to track multiple FM symptoms and physical functioning may improve treatment compliance as patients can also begin to appreciate their symptom evaluations improving over time. Such a tracking tool can also help to guide therapeutic decisions such as adding or removing medications, as well as recommending exercise, diet and/or cognitive behavioral strategies.

Realistic expectations from the healthcare provider's perspective communicated to the patient may also improve compliance. For example, the average patient improves by only 
$30-50 \%$ with monotherapy of any FDA-indicated FM agent. If greater improvement is to occur, patients will need to be active participants in their treatment by employing other evidence-based therapies such as lifestyle modification and exercise. However, many believe that reducing symptom burden first with appropriate pharmacotherapy will improve the likelihood that patients will be successful in the long-term adoption of nonpharmacologic treatments.

\section{Five-year view}

All three FDA-indicated FM drugs currently under review, including sodium oxybate, are tested as a monotherapy. In clinical practice, most drugs are given in combination. The longterm view of duloxetine in the treatment of FM will be shaped by studies that examine combination therapy, which reflects current clinical practice. Currently, clinical trials are in progress to look at these practices with commonly used FM medications such as duloxetine, tramadol, pregabalin and milnacipran (clinical trials registry numbers NCT01116531 and NCT00797797). These studies will shed light on current theoretical approaches that target various physiologic pathways theorized to be abnormal in FM.

Moreover, the long-term view will be influenced by data emerging from flexible dosing studies in duloxetine for FM (clinical trials registry number NCT00673452), which will facilitate a variety of dosing options, again, mirroring clinical practice. In addition, all of the aforementioned studies will report outcomes beyond pain, such as fatigue, mood, dyscognition and disordered sleep, which are all important symptoms that limit vocational and avocational activities in individuals with FM.

A final factor that will influence the long-term use of duloxetine for FM is that it will soon be the first of the FDA-indicated FM medications available in generic form, potentially becoming more affordable and acceptable to third-party payers. One concern with generic medications is that they are often priced per dose, and manufactured at up to $75 \%$ lower dose per unit. Since the indicated dose in FM is $60 \mathrm{mg}$, a generic may only be 20 or $30 \mathrm{mg}$, leading to potential subtherapeutic dosing. Unless the provider is aware of the appropriate dose to treat pain, the unfortunate results might be that the patient would not think that duloxetine provides effective pain relief.

As the safety data become more reassuring, the analgesic properties of duloxetine may expand, and in addition to the convenience of once-daily dosing, when used in combination, it may prove a valuable asset in the arsenal of chronic pain treatment.

\section{Acknowledgments}

Financial \& competing interests disclosure

This work was supported by NIH grants NINR (NR0007061), NCCAM (AT002688), NINR (NR0007061) and 1R21AR056751-01. In addition to NIH grant support, Kim D Jones receives grant support from Merck, Jazz and Forest Pharmaceuticals, and is on the Speaker Bureau for Pfizer, Lilly, Jazz and Forest Pharmaceuticals. In addition to NIH grant support, Rebecca L Ross is on the Speaker Bureau for Lilly and Jazz Pharmaceuticals.

No writing assistance was utilized in the production of this manuscript.

\section{References}

Papers of special note have been highlighted as:

•• of considerable interest 
1. Bennett RM. Clinical manifestations and diagnosis of fibromyalgia. Rheumatol. Dis. Clin. North Am 2009;35:215-232. • A good clinical review of fibromyalgia (FM) presentation.

2. Wolfe F, Clauw DJ, Fitzcharles M-A, et al. The American College of Rheumatology preliminary diagnostic criteria for fibromyalgia and measurement of symptom severity. Arthritis Care Res 2010;62:600-610.

3. Lawrence RC, Felson DT, Helmick CG, et al. Estimates of arthritis and other rheumatic conditions in the United States, Part II. Arthritis Rheum 2008;58:26-35. [PubMed: 18163497]

4. Annemans L, Le Lay K, Taieb C. Societal and patient burden of fibromyalgia syndrome. Pharmacoeconomics 2009;27:547-559. [PubMed: 19663526]

5. Silverman S, Dukes EM, Johnston SS, et al. The economic burden of fibromyalgia: comparative analysis with rheumatoid arthritis. Curr. Med. Res. Opin 2009;25:829-840. [PubMed: 19220165]

6. White LA, Birnbaum HG, Kaltenboeck A, et al. Employees with fibromyalgia: medical comorbidity, healthcare costs, and work loss. J. Occup. Environ. Med 2008;50:13-24. [PubMed: 18188077]

7. Wolfe F, Anderson J, Harkness D, et al. Work and disability status of persons with fibromyalgia. J. Rheumatol 1997;24:1171-1178. [PubMed: 9195528]

8. Wolfe F, Smythe HA, Yunus MB, et al. The American College of Rheumatology 1990 Criteria for the Classification of Fibromyalgia. Report of the Multicenter Criteria Committee. Arthritis Rheum 1990;33:160-172. [PubMed: 2306288]

9. Geisser ME, Gracely RH, Giesecke T, et al. The association between experimental and clinical pain measures among persons with fibromyalgia and chronic fatigue syndrome. Eur. J. Pain 2006;11:202-207. [PubMed: 16546424]

10. Staud R, Nagel S, Robinson ME, et al. Enhanced central pain processing of fibromyalgia patients is maintained by muscle afferent input: a randomized, double-blind, placebo-controlled study. Pain 2009;145:96-104. [PubMed: 19540671]

11. Ge H-Y, Nie H, Madeleine P, et al. Contribution of the local and referred pain from active myofascial trigger points in fibromyalgia syndrome. Pain 2009;147:233-240. [PubMed: 19819074]

12. Staud R. Biology and therapy of fibromyalgia: pain in fibromyalgia syndrome. Arthritis Res. Ther 2006;8(2):1-7. •• The latest theories for FM and other chronic pain syndromes.

13. Vierck JCJ. Mechanisms underlying development of spatially distributed chronic pain (fibromyalgia). Pain 2006;124:242-263. [PubMed: 16842915]

14. Williams DA, Gracely RH. Biology and therapy of fibromyalgia. Functional magnetic resonance imaging findings in fibromyalgia. Arthritis Res. Ther 2006;8(6):1-8.

15. Gracely RH, Geisser ME, Giesecke T, et al. Pain catastrophizing and neural responses to pain among persons with fibromyalgia. Brain 2004;127:835-843. [PubMed: 14960499]

16. Gracely RH, Petzke F, Wolf JM, et al. Functional magnetic resonance imaging evidence of augmented pain processing in fibromyalgia. Arthritis Rheum 2002;46:1333-1343. [PubMed: 12115241]

17. Harris RE, Sundgren PC, Pang Y-X, et al. Dynamic levels of glutamate within the insula are associated with improvements in multiple pain domains in fibromyalgia. Arthritis Rheum 2008;58:903-907. [PubMed: 18311814]

18. Petrou M, Harris RE, Foerster BR, et al. Proton MR spectroscopy in the evaluation of cerebral metabolism in patients with fibromyalgia: comparison with healthy controls and correlation with symptom severity. Am. J. Neuroradiol 2008;29:913-918. [PubMed: 18339723]

19. Landis CA, Lentz MJ, Rothermel J, et al. Decreased sleep spindles and spindle activity in midlife women with fibromyalgia and pain. Sleep 2004;27:741-750. [PubMed: 15283010]

20. Landis CA, Lentz MJ, Tsuji J, et al. Pain, psychological variables, sleep quality, and natural killer cell activity in midlife women with and without fibromyalgia. Brain Behav. Immun 2004;18:304313. [PubMed: 15157947]

21. Jones KD, Deodhar P, Lorentzen A, Bennett RM, Deodhar AA. Growth hormone perturbations in fibromyalgia: a review. Semin. Arthritis Rheum 2007;36:357-379. [PubMed: 17224178] 
22. McLean SA, Williams DA, Stein PK, et al. Cerebrospinal fluid corticotropin-releasing factor concentration is associated with pain but not fatigue symptoms in patients with fibromyalgia. Neuropsychopharmacology 2006;31:2776-2782. [PubMed: 16936702]

23. Wood PB, Glabus MF, Simpson R, et al. Changes in gray matter density in fibromyalgia: correlation with dopamine metabolism. J. Pain 2009;10:609-618. [PubMed: 19398377]

24. Wood PB, Ledbetter CR, Glabus MF, et al. Hippocampal metabolite abnormalities in fibromyalgia: correlation with clinical features. J. Pain 2009;10:47-52. [PubMed: 18771960]

25. Kuchinad A, Schweinhardt P, Seminowicz DA, et al. Accelerated brain gray matter loss in fibromyalgia patients: premature aging of the brain? J. Neurosci 2007;27:4004-4007. [PubMed: 17428976]

26. Cohen H, Neumann L, Glazer Y, et al. The relationship between a common catechol-Omethyltransferase (COMT) polymorphism val(158) met and fibromyalgia. Clin. Exp. Rheumatol 2009;27:S51-S56. [PubMed: 20074440]

27. Mease PJ, Choy EH. Pharmacotherapy of fibromyalgia. Rheumatol. Dis. Clin. North Am 2009;35:359-372. • A thorough review of medical management strategies for patients with FM.

28. Carville SF, Arendt-Nielsen S, Bliddal H, et al. EULAR evidence-based recommendations for the management of fibromyalgia syndrome. Ann. Rheum. Dis 2008;67:536-541. [PubMed: 17644548] •• An evidence-based overview of FM management.

29. Sarac AJ, Gur A. Complementary and alternative medical therapies in fibromyalgia. Curr. Pharm. Des 2006;12:47-57. [PubMed: 16454724]

30. Hassett AL, Gevirtz RN. Treatment for fibromyalgia: patient education, cognitive-behavioral therapy, relaxation techniques, and complementary and alternative medicine. Rheumatol. Dis. Clin. North Am 2009;35:393-407.

31. McVeigh JG, McGaughey H, Hall M, et al. The effectiveness of hydrotherapy in the management of fibromyalgia syndrome: a systematic review. Rheumatology Int 2008;29:119-130.

32. Jones KD, Liptan GL. Exercise interventions in fibromyalgia: clinical applications from the evidence. Rheumatol. Dis. Clin. North Am 2009;35:373-391.

33. Ang D, Chakr R, Mazzuca S, France CR, Steiner J, Stump T. Cognitive-behavioral therapy attenuates nociceptive responding in patients with fibromyalgia: a pilot study. Arthritis Care Res 2010;62:618-623.

34. Valencia M, Alonso B, Alvarez MJ, et al. Effects of 2 physiotherapy programs on pain perception, muscular flexibility, and illness impact in women with fibromyalgia: a pilot study. J. Manipulative Physiol. Ther 2009;32:84-92. [PubMed: 19121466]

35. Targino RA, Imamura M, Kaziyama HHS, et al. A randomized controlled trial of acupuncture added to usual treatment for fibromyalgia. J. Rehab. Med 2008;40:582-588.

36. Mayhew E, Ernst E. Acupuncture for fibromyalgia - a systematic review of randomized clinical trials. Rheumatology 2007;46:801-804. [PubMed: 17189243]

37. Babu AS, Mathew E, Danda D, et al. Management of patients with fibromyalgia using biofeedback: a randomized control trial. Indian J. Med. Sci 2007;61:455-461. [PubMed: 17679735]

38. Hassett AL, Radvanski DC, Vaschillo EG, et al. A pilot study of the efficacy of heart rate variability (HRV) biofeedback in patients with fibromyalgia. Appl. Psychophysiol. Biofeed 2007;32:1-10.

39. Boggio P, Amancia EJ, Correa CF, et al. Transcranial DC stimulation coupled with TENS for the treatment of chronic pain: a preliminary study. Clin. J. Pain 2009;25:691-695. [PubMed: 19920718]

40. Fregni F, Gimenes R, Valle AC, et al. A randomized, sham-controlled, proof of principle study of transcranial direct current stimulation for the treatment of pain in fibromyalgia. Arthritis Rheum 2006;54:3988-3998. [PubMed: 17133529]

41. Carette S, Bell MJ, Reynolds WJ, et al. Comparison of amitriptyline, cyclobenzaprine, and placebo in the treatment of fibromyalgia: a randomized, double-blind clinical trial. Arthritis Rheum 1994;37:32-40. [PubMed: 8129762] 
42. Mease PJ, Russell IJ, Arnold LM, et al. A randomized, double-blind, placebo-controlled, Phase III trial of pregabalin in the treatment of patients with fibromyalgia. J. Rheumatol 2008;35:502-514. [PubMed: 18278830]

43. Branco JC, Zachrisson O, Perrot S, et al. A European multicenter randomized double-blind placebo-controlled monotherapy clinical trial of milnacipran in treatment of fibromyalgia. J. Rheumatol 2010;37:851-859. [PubMed: 20156949]

44. Yalcin I, Tessier LH, Petit-Demoulière N, et al. $\beta 2$-adrenoceptors are essential for desipramine, venlafaxine or reboxetine action in neuropathic pain. Neurobiol. Dis 2009;33(3):386-394. [PubMed: 19084064]

45. Munro G. Dopamine d(1) and d920 receptor agonism enhances antinociception mediated by the serotonin and noradrenaline reuptake inhibitor duloxetine in the rat formalin test. Eur. J. Pharmacol 2007;575:66-74. [PubMed: 17725928]

46. Melzack R. From the gate to the neuromatrix. Pain 1999;6(Suppl.):S121-S126. [PubMed: 10491980]

47. Bonifazi M, Suman AL, Cambiaggi C, et al. Changes in salivary cortisol and corticosteroid receptor- $\alpha$ mRNA expression following a 3-week multidisciplinary treatment program in patients with fibromyalgia. Psychoneuroendocrinology 2006;31:1076-1086. [PubMed: 16962248]

48. McLean SA, Williams DA, Stein PK, et al. Cerebrospinal fluid corticotropin-releasing factor concentration is associated with pain but not fatigue symptoms in patients with fibromyalgia. Neuropsychopharmacology 2006;31:2776-2782. [PubMed: 16936702]

49. Li J, Simone DA, Larson AA. Windup leads to characteristics of central sensitization. Pain 1999;79(1):75-82. [PubMed: 9928779]

50. Rainville P, Bao Q,V, Chrétien P. Pain-related emotions modulate experimental pain perception and autonomic responses. Pain 2005;118(3):306-318. [PubMed: 16289802]

51. Arnold LM, Rosen A, Pritchett YL, et al. A randomized, double-blind, placebo-controlled trial of duloxetine in the treatment of women with fibromyalgia with or without major depressive disorder. Pain 2005;119:5-15. [PubMed: 16298061]

52. Perahia DGS, Pritchett YL, Desaiah D, Raskin J. Efficacy of duloxetine in painful symptoms: an analgesic or antidepressant effect? Int. Clin. Psychopharmacol 2006;21:311-317. [PubMed: 17012978]

53. Pedersen L, Nielsen A, Blackburn-Munro G. Antinociception is selectively enhanced by parallel inhibition of multiple subtypes of monamine transporters in rat models of persistent and neuropathic pain. Psychopharmacology 2005;182:551-561. [PubMed: 16133135]

54. Clauw DJ. Fibromyalgia: update on mechanisms and management. J. Clin. Rheumatol 2007;13(2): 102-109. [PubMed: 17414543] • Up-to-date theories on FM pathophysiology and management.

55. Chu LF, Clark DJ, Angst MS. Opioid tolerance and hyperalgesia in chronic pain patients after one month of oral morphine therapy: a preliminary prospective study. J. Pain 2006;7(1):43-48. [PubMed: 16414554]

56. Harris RE, Clauw DJ, Scott DJ, et al. Decreased central mu-opioid receptor availability in fibromyalgia. J. Neurosci 2007;27(37):10000-10006. [PubMed: 17855614]

57. Ahmedzai SH, Boland J. Opioids for chronic pain: molecular and genomic basis of actions and adverse effects. Curr. Opin Support. Palliat. Care 2007;1(2):117-125. [PubMed: 18685352]

58. Zucker DR, Ruthazer R, Schmid CH, et al. Lessons learned combining N-of-1 trials to assess fibromyalgia therapies. J. Rheumatol 2006;33(10):2069-2077. [PubMed: 17014022]

59. Russell IJ, Perkins AT, Michalek JE. Sodium oxybate relieves pain and improves function in fibromyalgia syndrome: a randomized, double-blind, placebo-controlled, multicenter clinical trial. Arthritis Rheum 2009;60(1):299-309. [PubMed: 19116896]

60. Stoll AL. Fibromyalgia symptoms relieved by flupirtine: an open-label case series. Psychosomatics 2000;41(4):371-372. [PubMed: 10906366]

61. Klawe C, Maschke M. Flupirtine: pharmacology and clinical applications of a nonopioid analgesic and potentially neuroprotective compound. Expert Opin. Pharmacother 2009;10(9):1495-1500.

[PubMed: 19505216] 
62. Wymer JP, Simpson J, Sen D, Bongardt S. Efficacy and safety of lacosamide in diabetic neuropathic pain: an 18-week double-blind placebo-controlled trial of fixed-dose regimens. Clin. J. Pain 2009;25(5):376-385. [PubMed: 19454870]

63. Younger J, Mackey S. Fibromyalgia symptoms are reduced by low-dose naltrexone: a pilot study. Pain Med 2009;10(4):663. [PubMed: 19453963]

64. Younger J, Zautra AJ, Cummins ET. Effects of naltrexone on pain sensitivity and mood in fibromyalgia: no evidence for endogenous opioid pathophysiology. PLoS Med 2009;4(4):e5180.

65. Sharma A, Goldberg MJ, Cerimele BJ. Pharmacokinetics and safety of duloxetine, a dual-serotonin and norepinephrine reuptake inhibitor. J. Clin. Pharmacol 2000;40:161-167. [PubMed: 10664922]

66. Trivedi MH, Desaiah D, Ossanna MJ, Pritchett YL, Brannan SK, Detke MJ. Clinical evidence for serotonin and norepinephrine reuptake inhibition of duloxetine. Int. Clin. Psychopharmacol 2008;23:161-169. [PubMed: 18408530]

67. Skinner MH, Kuan H-Y, Pan A, et al. Duloxetine is both an inhibitor and substrate of cytochrome P4502D6 in healthy volunteers. Clin. Pharmacol. Ther 2003;73(3):170-177. [PubMed: 12621382]

68. Spina E, Santoro V, D'Arrigo C. Clinically relevent pharmacokinetic drug interactions with second-generation antidepressants: an update. Clin. Ther 2008;30(7):1206-1227. [PubMed: 18691982]

69. Lobo ED, Quinlin T, O’Brien L, Knadler MP, Heathman M. Population pharmacokinetics of orally administered duloxetine in patients. Clin. Pharmacokinet 2009;48(3):189-197. [PubMed: 19385712]

70. Choy EHS, Mease PJ, Kajdasz DK, et al. Safety and tolerability of duloxetine in the treatment of patients with fibromyalgia: pooled analysis of data from five clinical trials. Clin. Rheumatol 2009;28:1035-1044. [PubMed: 19533210]

71. Lobo ED, Loghin C, Knadler MP, et al. Pharmacokinetics of duloxetine in breast milk and plasma of healthy postpartum women. Clin. Pharmacokinetics 2008;47(2):103-109.

72. Chan C, Yeo KP, Pan AX, Lim M, Knadler MP, Small DS. Duloxetine pharmacokinetics are similar in Japanese and Caucasian subjects. Br. J. Clin. Pharmacol 2006;63(3):310-314. [PubMed: 17380590]

73. Gahimer J, Wernicke J, Yalcin I, Ossanna MJ, Wulster-Radcliffe M, Viktrup L. A retrospective pooled analysis of duloxetine safety in 23,983 subjects. Curr. Med. Res. Opin 2007;23(1):175184. [PubMed: 17257478]

74. Lantz RJ, Gillespie TA, Rash TJ, Kuo F, Skinner H-Y, Knadler MP. Metabolism, excretion and pharmacokinetics of duloxetine in healthy human subjects. Drug Metab. Dispos 2003;31(9):11421150. [PubMed: 12920170]

75. Arnold LM, Lu Y, Crofford LJ, et al. A double-blind, multicenter trial comparing duloxetine with placebo in the treatment of fibromyalgia patients with or without major depressive disorder. Arthritis Rheum 2004;50:2974-2984. [PubMed: 15457467]

76. Russell IJ, Mease PJ, Smith TR, et al. Efficacy and safety of duloxetine for treatment of fibromyalgia in patients with or without major depressive disorder: results from a 6-month randomized, double-blind, placebo-controlled, fixed-dose trial. Pain 2008;136:432-444. [PubMed: 18395345]

77. Chappell AS, Bradley L, Wiltse C, et al. A six-month double-blind, placebo-controlled, randomized clinical trial of duloxetine for the treatment of fibromyalgia. Int. J. Gen. Med 2008;1:91-102. [PubMed: 20428412]

78. Chappell AS, Littlejohn G, Kajdasz DK, et al. 1-year safety and efficacy study of duloxetine in patients with fibromyalgia. Clin. J. Pain 2009;25(5):365-375. [PubMed: 19454869]

79. Farrar JT, Young JP Jr, LaMoreaux L, et al. Clinical importance of changes in chronic pain intensity measured on an 11-point numerical pain rating scale. Pain 2001;94:149-158. [PubMed: 11690728]

80. Argoff CE, Misha-Miroslav B, Belgrade MJ, et al. Consensus guidelines: treatment planning and options. Mayo Clin. Proc 2006;81(4):S12-S25. [PubMed: 16608049]

81. Arnold LM, Pritchett YL, D'Souza DN, et al. Duloxetine for the treatment of fibromyalgia in women: pooled results from two randomized, placebo-controlled clinical trials. J. Womens Health 2007;16(8):1145-1156. 
82. Arnold LM, Hudson JI, Wang F. Comparisons of the efficacy and safety of duloxetine for the treatment of fibromyalgia in patients with versus without major depressive disorder. Clin. J. Pain 2009;25:461-468. [PubMed: 19542792]

83. Bradley LA, Wohlreich MM, Wang F, et al. Pain response profile of patients with fibromyalgia treated with duloxetine. Clin. J. Pain 2010;26:498-504. [PubMed: 20551724]

84. Lunn MPT, Hughes RAC, Wiffen PJ. Duloxetine for treating painful neuropathy or chronic pain (review). Cochrane Database Syst. Rev 2010;4 CD007115.

85. Duloxetine: new indication. Depression and diabetic neuropathy: too many adverse effects. Prescrire Int 2006;15(85):168-172. [PubMed: 17121211]

86. Duloxetine (cymbalta) for fibromyalgia. Med. Lett. Drugs Ther 2008;50(1291):57-58. No authors listed.

87. Spielmans GI. Duloxetine does not relieve painful physical symptoms in depression: a metaanalysis. Psychother. Psychosomat 2008;77(1):12-16.

88. Sclar D, Robison LM, Skaer TL. Concomitant triptan and SSRI or SNRI use: a risk for serotonin syndrome. Headache 2008;48(1):126-129. [PubMed: 18005139]

89. Wernicke J, Lledo A, Raskin J, Kajdasz DK, Wang F. An evaluation of the cardiovascular safety profile of duloxetine. Drug Safe 2007;30(5):437-455.

90. McCloskey D, Postoloche TT, Vittone BJ, et al. Selective serotonin reuptake inhibitors: measurement of effect on platelet function. Trans. Res. J. Lab Clin. Med 2008;151(3):168-172.

91. Loke Y, Trivedi AN, Singh S. Meta-analysis: gastrointestinal bleeding due to interaction between selective serotonin uptake inhibitors and non-steroidal anti-inflammatory drugs. Aliment. Pharmacol. Ther 2008;27(1):31-40. [PubMed: 17919277]

92. Schalekamp T, Klungel OH, Souverein PC, de Boer A. Increased bleeding risk with concurrent use of selective serotonin reuptake inhibitors and coumarins. Arch. Int. Med 2008;168(2):180-185. [PubMed: 18227365]

93. Gibbons R, Hur K, Bhaumik DK, Mann JJ. The relationship between antidepressant prescription rates and rate of early adolescent suicide. Am. J. Psychiatry 2006;163(11):1898-1904. [PubMed: 17074941]

94. Juurlink D, Mamdani MM, Kopp A, Redelmeier DA. The risk of suicide with selective serotonin reuptake inhibitors in the elderly. Am. J. Psychiatry 2006;163(5):813-821. [PubMed: 16648321]

95. Benvenuti A, Rucci P, Miniati M, et al. Treatment-emergent mania/hypomania in unipolar patients. Bipolar Dis 2008;10(6):726-732.

96. Arnold LM, Russell IJ, Diri EW, et al. A 14-week, randomized, double-blinded, placebo-controlled monotherapy trial of pregabalin in patients with fibromyalgia. J. Pain 2008;9(9):792-805. [PubMed: 18524684]

97. Mease PJ, Clauw DJ, Gendreau RM, et al. The efficacy and safety of milnacipran for treatment of fibromyalgia. a randomized, double-blind, placebo-controlled trial. J. Rheumatol 2009;36(2):398409. [PubMed: 19132781]

\section{References}

\section{Websites}

101. Globe Newswire. Pipex Pharmaceuticals' Oral Flupirtine Receives IRB Approval to Initiate Phase II Clinical Trial for Fibromyalgia. [Accessed 10 April 2010]. www.globenewswire.com/newsroom/news.html?ref=rss\&d=151691

102. FDA. Introduction to duloxetine. FY 2004 PDUFA Performance Report. [Accessed 30 June 2010]. www.fda.gov/AboutFDA/ReportsManualsForms/Reports/UserFeeReports/PerformanceReports/ PDUFA/ucm115035.htm\#APPENDIXB:ListofApprovedApplications

103. Drugs @ FDA. [Accessed 30 June 2010]. 2009 www.accessdata.fda.gov/drugsatfda_docs/label/2009/021427s030lbl.pdf

104. FDA Drug Safety Newsletter. [Accessed 30 June 2010]. www.fda.gov/cder/dsn/default.htm 
105. FDA. Postmarketing Drug Safety Information for Patients and Providers. Duloxetine (marketed as Cymbalta) information. [Accessed 30 June 2010].

www.fda.gov/Drugs/DrugSafety/PostmarketDrugSafetyInformationforPatientsandProviders/ ucm114966.htm

106. Index of FIQ. www.myalgia.com/FIQ 


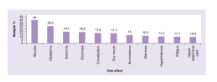

Figure 1. Most common side effects of duloxetine dosed at 60-120 mg per day Data from [78]. 


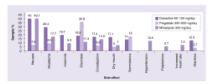

Figure 2. Comparisons of the most common side effects of the three US FDA-approved drugs for fibromyalgia

Data from $[78,96,97]$. 


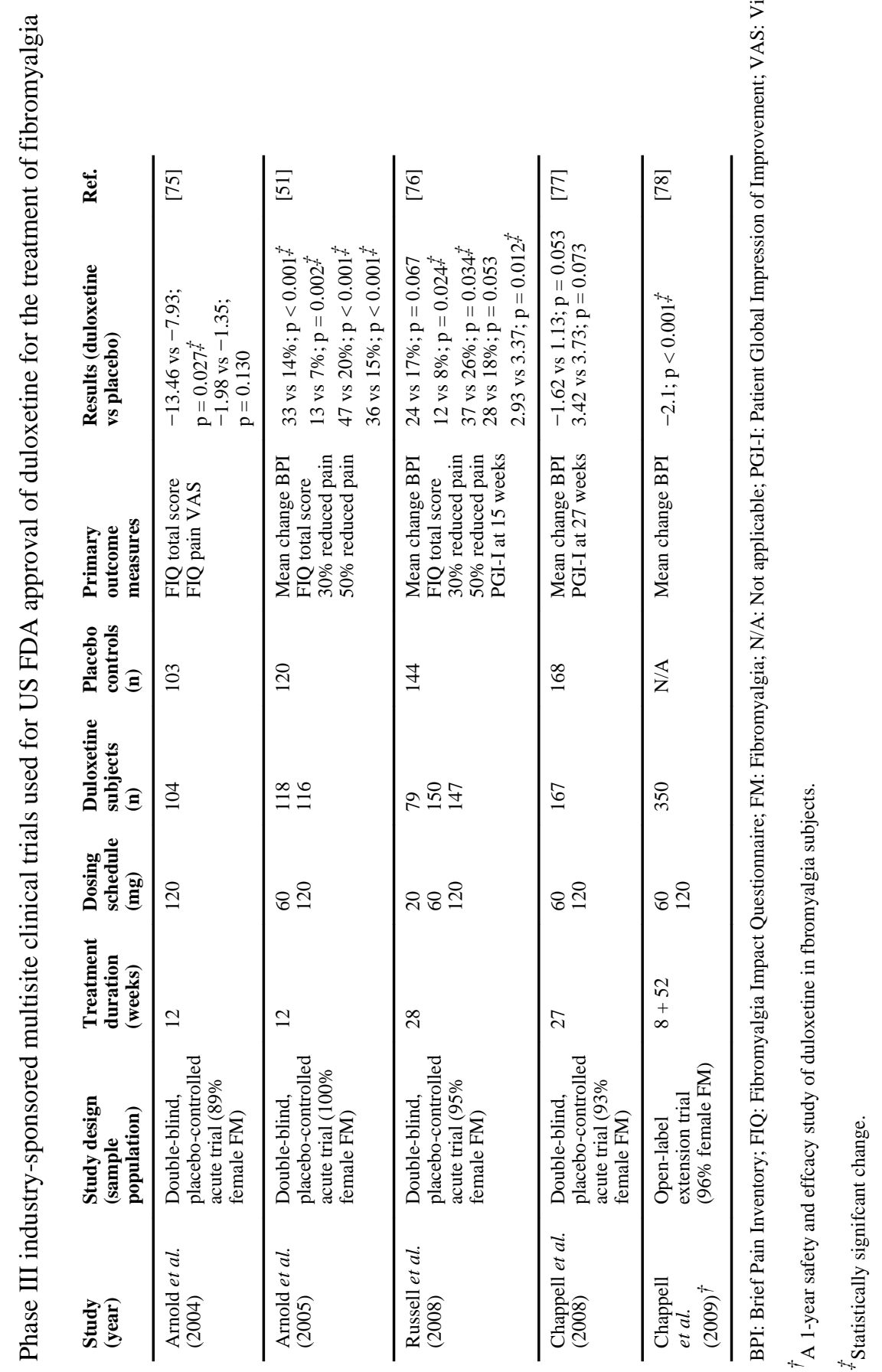

